\title{
Chiari malformation in adults: relation of morphological aspects to clinical features and operative outcome
}

\author{
J M Stevens, W A D Serva, B E Kendall, A R Valentine, J R Ponsford
}

\begin{abstract}
To determine whether clinical features attributed to cerebellar ectopia could be related to the severity of the malformation, and if morphological features could be related to operative outcome, a retrospective study of 141 patients with the adult Chiari malformation was carried out, 81 receiving operative treatment. Morphological parameters derived from preoperative clinical imaging were compared with presenting clinical features and postoperative outcomes. Patients with the most severe cerebellar malformation, defined as descent of the cerebellar tonsils to or below the axis, had disabling ataxia and nystagmus more frequently. Those with brainstem compression had limb weakness and muscle wasting more frequently. Operative outcome was significantly less favourable in patients with severe cerebellar ectopia (12\% improved, 69\% deteriorated) than in those with minor ectopia (50\% improved, $17 \%$ deteriorated). Patients with a distended cervical syrinx had a more favourable outcome than those without. Morphological features help predict operative risk.
\end{abstract}

$(\mathcal{F}$ Neurol Neurosurg Psychiatry 1993;56:1072-1077)

The term adult Chiari malformation is used to describe descent of the hind brain into the cervical canal, in which meningomyelocoele is absent and hydrocephalus rare, and patients usually present in adult life. ${ }^{1}$ The clinical features which may be associated are protean ${ }^{2-6}$ and a causal relationship in a given case may not be established with certainty, especially as modern computed imaging suggests that minimal protrusion of parts of the cerebellar hemispheres through the foramen magnum is relatively common, and that even marked protusion is often asymptomatic. ${ }^{7}$ The issue is important because it is often maintained that disability due to the adult Chiari malformation usually is curable by operation. ${ }^{15}$

In the nine operative series which have included more than 20 patients and in which the mean follow up was at least 6 months, the mean proportion and patients with sustained improvement has been $56 \%$ (range $29-82 \%$ ), the proportion who deteriorated $24 \%$ (range $6-31 \%)$ and the perioperative mortality $3 \%$ (range $0-5 \cdot 6 \%) .{ }^{128-14}$ The clinical features most likely to be alleviated have been headache and neck pain, and those most refractory were often ataxia and nystagmus.

Clearly not all patients with the adult Chiari malformation benefit from operation. Attempts at risk stratification have been made, and recently a careful study by Menezes' group ${ }^{14}$ established three preoperative clinical features predictive of a poor operative outcome-namely, ataxia, scoliosis, and muscle atrophy. Attempts at relating the severity of the Chiari lesion to individual clinical features or syndromes have been few and some of the conclusions conflicting. ${ }^{15-17}$ There has been no formal attempt at operative risk stratification based on morphological features.

The present study was begun several years ago to investigate if the severity of the hind brain abnormality could be related to preoperative clinical features and postoperative outcome. Because the design of the investigation was retrospective, and large numbers of patients with detailed clinical data were required, it was necessary to use data mainly from myelography and CT rather than MRI which only became available at our institution some time after the study began. Nevertheless, most of the criteria used in the study are equally applicable to MRI.

\section{Materials and methods}

The study consisted of a retrospective review of 141 symptomatic adult patients diagnosed as having a Chiari malformation by myelography alone in 91 or in combination with CT in 50 patients. At least five of these patients also had MRI performed on a Vista MR (Picker International) operating at 0.5 or $0.26 \mathrm{~T}$. One patient only had hydrocephalus, and none had a meningomyelocoele. Eighty one patients were operated on in one of three surgical units; all were subjected to a posterior suboccipital and upper cervical decompression, 12 had partial excision of herniated cerebellar tonsils, and eight had an occlusion of the obex. Thirty one patients also had a syringostomy. Sixty patients did not receive operations at our institution: 32 were lost to follow up and may have been operated on elsewhere, 18 had significant co-existing disease such as multiple sclerosis (10 patients) and severe spondylotic spinal cord compression (eight patients), eight refused operation, and it was unclear why no operation was performed in two.

Clinical data were gleaned from the case notes and evaluated by one of two neurolo- 
Table 1 Clinical groupings.

(a) General

Headache: considered only if it was exacerbated by head movement, exercise, or coughing.

Drop attacks: unexplained falls with or without brief loss of consciousness.

3 Neck pain: with or without occipital radiation.

(b) Functional anatomical

Upper and brainstem (midbrain, pons): dysfunction of cranial nerves III, IV, V, VI, VII, and VIII.

2 Medulla oblongata: dysfunction of cranial nerves IX, X, XI, XII.

3 Brainstem - cerebellar connections: ataxia, vertigo, nystagmus.

4 Peripheral sensory disturbances: impaired pain and temperature sensation; loss of position and vibratory sense; subjective complaints of numbness of parasthesiae in limb or trunk.

5 Peripheral motor disturbances: all forms of weakness in limbs or trunk, with or without muscle wasting. Syringomyelia: this category included only those patients in which there was a firm diagnosis of syringomyelia in the case notes, based on clinical and radiological findings.

(c) Postoperative clinical categories

1 Original clinical features: The preoperative symptoms signs were classified postoperatively as either improved, unchanged or worse.

2 New clinical features: any complaints or signs appearing after operation which was not present before, excluding those directly related to the wound itself.

3 Headache: this feature was considered separately when present in the preoperative clinical picture.

gists (WS and JP) not involved in the routine management of the patients. A large volume of data was obtained which, for the purposes of analysis, was simplified by arranging it into the categories and groups indicated in table 1. Missing data in any individual case were recorded as such, and the case excluded from analysis of that feature. Data sets were judged to be adequate for 127 patients. The postoperative assessments were divided into early and late. All early assessments were made within 1 month of operation and features referable to the surgical wound were excluded, such as neck pain and stiffness. The late assessments were made, as far as possible, from the last outpatient attendance. This varied from 3 months to 9 years after operation; but in only 18 did this period exceed 2 years and the mean follow up period was 1.2 years. The available non-operated group was considered too small and heterogeneous to be used for a meaningful parallel group follow up study.

The radiological features were assessed by three experienced neuroradiologists (JS, BK, AV).

\section{CEREBELLAR TONSILS}

The level of the cerebellar tonsils was assessed with head and spine either slightly extended or slightly flexed but never hyperflexed. The distance the most inferior part of one or both cerebellar hemispheres descended below the foramen magnum was measured in millimetres on all available images, and the level also was noted relative to easily recognisable anatomical landmarks. Magnification was not adequately standardised between examination types, so only the relative levels, reasonably consistent between myelography, computed myelography, and MRI were considered in the final analysis. Patients were grouped into three categories: $A$, tonsils between foramen magnum and arch of the atlas; $B$, tonsils between the arches of atlas and axis; and C, tonsils below the axis. The upper border of each neural arch was taken as the reference point for each group.

\section{FORAMEN MAGNUM}

The degree of obstruction at the foramen magnum was also assessed. Contrast medium flowed freely into the head from the spinal subarachnoid space via the anterior cerebrospinal fluid pathway on myelography in all cases, although in some it was obstructed posteriorly. Two types of observation were made:

1 The size of the cisterna magna was assessed both above and at the level of the foramen magnum and its mid-sagittal size determined by measuring the minimum distance between the internal occipital crest and posterior margin of the foramen magnum and the cerebellum. CT or midline sagittal tomograms and MRI were used wherever possible for this assessment. Accurate measurement was not possible, but the cisterna magna was scored as obliterated if it was not visible, small if its mid-sagittal width was less than $2-3 \mathrm{~mm}$, and normal if it was greater than $2-3 \mathrm{~mm}$.

2 Contrast medium visible in the fourth ventricle either during the myelogram, or on CT within 1 hour of the myelogram provided concentration was similar to that in the posterior fossa cisterns, was recorded. This was taken as evidence that either one or each of the foramena of Magendie or of Lushka were patent. Arachnoiditis was considered present if the cisterna magna was obliterated and the fourth ventricle failed to opacify on computed myelography.

\section{SYRINGOMYELIA}

The upper spinal cord was also assessed for the presence of syringomyelia. Syringomyelia was considered probable if there was a central accumulation of contrast medium within the spinal cord on early or delayed postmyelography CT, or a circumscribed central signal change was visible on MRI, or if there was a diffuse abnormality of cord size not explained by compression. A mid-sagittal diameter of the spinal cord of greater than 10 $\mathrm{mm}$ was regarded as definitely enlarged, and of less than $6 \mathrm{~mm}$ as definitely small. Measurements between were regarded as normal.

\section{MEDULLA OBLONGATA}

Compression of the medulla oblongata was also considered. This was often difficult to assess on myelography, and only assessments made on adequate computed cisternograms and MRI were analysed. The anterior and posterior surfaces were considered separately, and compression was considered present when the whole or part of the contour was flattened or concave on axial images. The posterior surface of the medulla was assessed below the obex because the floor of the fourth ventricle usually is concave. The compressing agent also was recorded, and was either the apex of the dens, an abnormality of the clivus, or the cerebellar tonsils acting either alone or 
Table 2 Clinical features in 141 patients *

\begin{tabular}{lll}
\hline & Symptoms & Signs \\
\hline Headache & 41 & - \\
Neck pain & 46 & - \\
Drop attacks & 19 & - \\
Pons-midbrain & 27 & 44 \\
Medulla oblongata & 49 & 61 \\
Brainstem/cerebellar & 48 & 73 \\
Peripheral sensory & 67 & 67 \\
Peripheral motor & 33 & 92 \\
\hline *Patients often had more than one clinical feature and
\end{tabular}

Patients often had more than one clinical feat
adequate data sets were available in only 127 patients.

Table 3 Postoperative clinical assessments

\begin{tabular}{|c|c|c|c|c|}
\hline & \multicolumn{2}{|l|}{ Early } & \multicolumn{2}{|l|}{ Late } \\
\hline & $n$ & $\%$ & $n$ & $\%$ \\
\hline \multicolumn{5}{|c|}{ Original Features: } \\
\hline Improved* & 20 & 25 & 23 & 34 \\
\hline Unchanged & 31 & 38 & 19 & 28 \\
\hline Worse & 30 & 37 & 25 & 37 \\
\hline Total & 81 & 100 & 67 & 100 \\
\hline New features: & $23 / 81$ & 28 & $21 / 67$ & 31 \\
\hline \multicolumn{5}{|l|}{ Headache: } \\
\hline Improved & 21 & 68 & 17 & 63 \\
\hline Unchanged & 1 & 13 & 3 & 11 \\
\hline Worse & 9 & 29 & 7 & 26 \\
\hline Total & 31 & 100 & 27 & 100 \\
\hline
\end{tabular}

ॠThis implies that one or more cardinal features showed definite improvement; usually it was only one. No case appeared to
have improved in one direction and deteriorated in another.

in combination. Compression was graded visually as mild, moderate, or severe - severe indicating that the mid-sagittal diameter of the neural axis at the point of maximal compression was reduced by $50 \%$ or more.

All the clinical and radiological features were assigned a numerical code and the data were analysed using the Statistical Package for the Social Sciences (SPSS) version 7 program, on the University of London Computer Centre Amdahl Computer. The incidence of each clinical feature was compared with the presence or absence of each radiological feature, considered independently. In some instances a relationship was sought between individual radiological features, but the analysis was concerned mainly with relationship of radiological to clinical features, especially operative outcome. $\chi^{2}$ tests of association were used, except when an expected frequency was less than five, in which case a Fisher's exact test was used.

Table 4 Clinical features related to tonsillar descent

\begin{tabular}{|c|c|c|c|c|}
\hline & \multicolumn{4}{|c|}{ Level of tonsils* } \\
\hline & $(0-C 1)$ & $(C 1-C 2)$ & $(>C 2)$ & p Value \\
\hline Medulla (symptoms) & $\begin{array}{l}16 / 53 \\
(30 \%)\end{array}$ & $\begin{array}{l}14 / 49 \\
(29 \%)\end{array}$ & $\begin{array}{l}14 / 25 \\
(56 \%)\end{array}$ & 0.04 \\
\hline Peripheral motor (sign) & $\begin{array}{l}28 / 53 \\
(53 \%)\end{array}$ & $\begin{array}{l}32 / 49 \\
(65 \%)\end{array}$ & $\begin{array}{l}21 / 25 \\
(84 \%)\end{array}$ & 0.02 \\
\hline $\begin{array}{l}\text { Brainstem/Cerebellar } \\
\text { (signs) }\end{array}$ & $\begin{array}{l}19 / 53 \\
(36 \%)\end{array}$ & $\begin{array}{l}26 / 49 \\
(53 \%)\end{array}$ & $\begin{array}{l}18 / 25 \\
(72 \%)\end{array}$ & 0.01 \\
\hline $\begin{array}{l}\text { Peripheral sensory } \\
\text { (symptoms) }\end{array}$ & $\begin{array}{l}29 / 53 \\
(55 \%)\end{array}$ & $\begin{array}{l}27 / 49 \\
(53 \%)\end{array}$ & $\begin{array}{l}6 / 24 \\
(25 \%)\end{array}$ & 0.03 \\
\hline Neck pain & $\begin{array}{l}24 / 53 \\
(47 \%)\end{array}$ & $\begin{array}{l}11 / 48 \\
(23 \%)\end{array}$ & $\begin{array}{l}5 / 25 \\
(20 \%)\end{array}$ & 0.02 \\
\hline Syringomyelia & $\begin{array}{l}28 / 49 \\
(57 \%)\end{array}$ & $\begin{array}{l}31 / 44 \\
(70 \%)\end{array}$ & $\begin{array}{l}6 / 25 \\
(20 \%)\end{array}$ & 0.00 \\
\hline
\end{tabular}

${ }^{\star} 0-\mathrm{C} 1$, tonsils between occiput and the atlas ring; $\mathrm{C} 1-2$, tonsils between the atlas and axis; $>\mathrm{C} 2$, tonsils below the axis.

\section{Results}

The five radiological variables were tested for an association with each of the 15 clinical variables which included both early and late postoperative assessments. There were 75 sets of contingency tables of which 17 showed significant associations (24\%). In addition, the six postoperative clinical variables in the early and late assessments were compared with each of the preoperative clinical variables, to create a further 90 contingency tables, of which only four indicated significant associations (4\%).

The duration of symptoms was less than 5 years in $46 \%$ and more than 10 years in $26 \%$. Patients' ages ranged from 11 to 78 years, but $54 \%$ were aged between 32 and 45 years. The distribution of preoperative clinical features in the sample is shown in table 2, although it is noted that adequate sets were available in only 127 patients. The outcomes in the 81 operated patients are indicated in table 3. However, adequate sets of clinical and radiological data were available for the early postoperative assessment in only 73, and in 60 for the late assessment. Headache was included only if specifically mentioned in the postoperative notes, so that only 31 cases had valid early assessments, and only 27 valid late assessments. Sixty five patients had radiological evidence of syringomyelia (55\%). Basilar invagination or other significant bony abnormalities were recognised in 17 cases.

Associations between several clinical features and radiological parameters which yielded probability values less than 0.05 are indicated in tables 4 and 5. In addition, an enlarged spinal cord was associated with the presence of peripheral sensory disturbances which were present in $73 \%$ of 63 valid cases in whom the cord was enlarged, and in only $17.5 \%$ of 40 valid cases in whom the cord was normal or small $(p=0.0001)$. It was not possible to investigate the effect of a small spinal cord as opposed to a normal cord, since the numbers recorded as having a small cord were too small. Similarly there was a strong positive association between abnormal contrast accumulation in the cord on postmyelography CT and peripheral sensory disturbance ( 21 of 28 valid cases with contrast in the cord had sensory disturbance compared with seven of 22 without, $p=0.002$ ). Interestingly, syringomyelia showed no association with obliteration of the cisterna magna or opacification of the fourth ventricle $(p=0 \cdot 130)$.

A significant association was found between operative outcome, and the level of cerebellar tonsils (table 6). A further association was found between clinical improvement and the presence of an enlarged spinal cord

Table 5 Clinical features related to size of cisterna magna

\begin{tabular}{|c|c|c|c|c|}
\hline & Normal & Small & Obliterated & p Value \\
\hline $\begin{array}{l}\text { Medulla oblongata } \\
\text { (signs) }\end{array}$ & $\begin{array}{l}5 / 18 \\
(28 \%)\end{array}$ & $\begin{array}{l}31 / 74 \\
(42 \%)\end{array}$ & $\begin{array}{l}10 / 12 \\
(83 \%)\end{array}$ & 0.02 \\
\hline $\begin{array}{l}\text { Brainstem-cerebellar } \\
\text { (signs) }\end{array}$ & $\begin{array}{l}3 / 18 \\
(16 \%)\end{array}$ & $\begin{array}{l}39 / 74 \\
(53 \%)\end{array}$ & $\begin{array}{l}10 / 12 \\
(83 \%)\end{array}$ & 0.00 \\
\hline
\end{tabular}


Table 6 Operative outcome with respect to level of tonsils

\begin{tabular}{|c|c|c|c|c|c|c|}
\hline \multirow[b]{2}{*}{$\begin{array}{l}\text { Preoperative } \\
\text { clinical features }\end{array}$} & \multicolumn{6}{|c|}{ Level of tonsils } \\
\hline & $\begin{array}{l}(0-C 1) \\
n\end{array}$ & $\%$ & $\begin{array}{l}(C 1-C 2) \\
n\end{array}$ & $\%$ & $\begin{array}{l}(>C 2) \\
n\end{array}$ & $\%$ \\
\hline \multicolumn{7}{|l|}{ Early: } \\
\hline Worse & 5 & 18 & 14 & 47 & 11 & 69 \\
\hline Unchanged & 13 & 48 & 11 & 37 & 3 & 19 \\
\hline Improved & 9 & 33 & 5 & 17 & 2 & 12 \\
\hline Total & 27 & 100 & 30 & 100 & $1 \overline{6}$ & 100 \\
\hline \multicolumn{7}{|l|}{ Late: } \\
\hline Worse & 3 & 17 & 7 & 26 & 9 & 60 \\
\hline Unchanged & 6 & 33 & 7 & 26 & 4 & 27 \\
\hline Improved & 9 & 50 & 13 & 48 & 2 & 13 \\
\hline Total & 18 & 100 & 27 & 100 & 15 & 100 \\
\hline
\end{tabular}

By combining the categories of unchanged and improved, $p$ values were calculated as 0.02 for early and 0.04 for late assessment.

on early $(p=0.01)$ but not on late $(p=$ 0.082 ) assessment (table 7).

The question of arachnoiditis could not be addressed directly in this study. The combination of obliteration of the cisterna magna and non-filling of the fourth ventricle may have been due to arachnoiditis in many cases. Both were associated with the cerebellar tonsils lying below $\mathrm{C} 2$, but only non-filling of the fourth ventricle showed a relationship with poor operative outcome. When the fourth ventricle was opacified, two of 15 patients were worse after operation compared with 28 of 50 when the fourth ventricle did not opacify $(p=0.001)$. The role of arachnoiditis as opposed to apparent level of the cerebellar tonsils could not be established by this analysis.

Post-myelography CT studies all too frequently were of limited extent, and often delayed until the contrast density in the spinal and intracranial subarachnoid spaces was much reduced, preventing detailed analysis. At the time the potential value of detailed assessment was not adequately appreciated, a situation which this study originally was designed to redress. Therefore, only 28 cases permitted what we now regard as an adequate estimation of medullary compression. The majority of attempted clinical associations with compression failed to suggest any association but of the 21 who had some compression 18 had peripheral motor signs (mainly weakness and wasting), and only three of seven without compression had such signs ( $p$ $=0.043$, Fisher's exact test). Only 17 cases were suitable for analysis of operative outcome, and although five patients with mild compression were unchanged, whereas nine of 12 with moderate or severe compression suffered a deterioration in original signs and three also developed new features, a Fisher's exact test indicated a $p$ value of 0.06 which was not significant. The major compressing agent was judged to be anterior in 18 (dens

Table 7 Operative outcome ${ }^{\star}$ with respect to spinal cord size

\begin{tabular}{llrlll}
\hline \multirow{2}{*}{$\begin{array}{l}\text { Preoperative clinical } \\
\text { features (early) }\end{array}$} & \multicolumn{2}{l}{ Normal } & & \multicolumn{2}{l}{ Enlarged } \\
\cline { 2 - 3 } \cline { 5 - 6 } & $n$ & $\%$ & & \multicolumn{1}{c}{$\%$} \\
\hline Worse & 10 & 59 & & 8 & 23 \\
Unchanged/better & 7 & 41 & & 28 & 82 \\
Total & 17 & 100 & & 34 & 100 \\
\hline
\end{tabular}

^Based on early assessment $(p=0.01)$.
17, clivus 1) and posterior in three (cerebellar tonsils).

Analysis of other aspects of medullary morphology, such as length, level of obex and dorsal column nuclei, presence of spur or kink, were possible in only 16 cases with computed cisternograms and the five cases who also had MRI. In seven there was a cervicomedullary spur just below the ectopic cerebellar tonsils over which the distal part of the fourth ventricle was prolonged, the obex presumably lying under the spur. In all seven the cerebellar tonsils were at or below $\mathrm{C} 2$ (one was in group B, and six were in group C). There were no independent clinical features distinguishing this small group of patients, though none had syringomyelia.

As would be expected from previous findings, poor operative outcome showed a relationship with the presence of swallowing difficulties, truncal ataxia, and nystagmus ( $p$ $=0.025$ in each instance), all of which had shown an association with marked tonsillar descent. Patient age and duration of clinical symptoms were considered, and no significant association was found with operative outcome on two sample $t$-tests. Mean ages in the worse, unchanged, and improved groups were $41.6,41 \cdot 1$, and 32.0 years; and mean duration of symptoms in the same groups were $5 \cdot 6,4 \cdot 1$, and $4 \cdot 5$ years.

\section{Discussion}

The distribution of clinical features indicated that this sample of patients was similar to that in other comparable studies, and the results of subocciptal decompression, when due allowance was made for varying methods of assessment, were generally compatible with the consensus of published reports already cited. The mean follow up was relatively short, many other series having mean periods of more than 2 years. The group of operated patients with mild cerebellar tonsillar descent (group A), who generally fared best, were most frequently lost to follow up. Although the proportion of positive associations was similar to that which might have been expected by chance, the positive findings were broadly in accord with those of Elster et $a l^{17}$ and Dyste et $a l^{14}$ and, where relevant, showed the interrelationships expected from true as opposed to spurious associations. For example, marked tonsillar descent was associated with the preoperative ataxia and poor operative outcome, and poor operative outcome was associated with preoperative ataxia.

The most frequent relationships involved the level of the cerebellar tonsils. It has been observed at operation that sometimes the tonsils are found at a lower level than suggested by myelography. ${ }^{219}$ Possibly the altered hydrodynamics at the craniovertebral junction caused by opening the dura explains at least some such discrepancies, and hyperflexion of the head may explain others. MRI is now the imaging method of choice and how estimates of tonsillar descent compared with those from myelography in the same group of patients is unknown. The measurements 
made by Aboulezz et $a l^{7}$ on MRI compared with those made from myelography by O'Connor et $a l^{20}$ and Baker, ${ }^{21}$ suggest that they appear slightly lower on MRI. Observations from high definition computed cisternography have indicated that the medial parts of the biventral lobules are nearly always inferior to the tonsils in normal cerebellar hemispheres and lie in the plane of the foramen magnum posterolateral to the brainstem, very occasionally protruding slightly below. ${ }^{22}$ Only when ectopic do the cerebellar tonsils enter the foramen magnum or protrude below it, usually lying directly posterior to the brainstem. Most MRI assessments are made from mid-sagittal images usually 3 to $5 \mathrm{~mm}$ thick in which this distinction often cannot reliably be made. It is even more difficult on myelography unless thin section tomography is used. In our cases, all that could reliably be said was that some part of the cerebellar hemispheres was visible in the cervical part of the cisterna magna. In the cases with CT, protrusion of the actual cerebellar tonsils could be confirmed and the relative degree of protrusion correlated reasonably on MRI and myelographic assessment.

Computed cisternography and MRI indicate that patients with herniated cerebellar tonsils often have an elongated and sometimes even kinked medulla oblongata in which the obex lies below the foramen magnum, ${ }^{23}{ }^{25}$ as was the case in at least seven of our patients and probably also in many more. In many publications such a configuration would be classified as Chiari type II. ${ }^{13-1724}$ The association between increasing tonsillar descent and obliteration or smallness of the cisterna magna probably accounted for the low incidence of filling of the fourth ventricle in group C. However, it was less clear why more severe tonsillar descent should have been associated with a significantly low incidence of syringomelia. The explanation may reside in the significance of abnormalities of the medulla oblongata such as the kink and spur, the incidence of which is increased in patients with more severe malformations. ${ }^{25}$ Such morphology is associated with a lower incidence of syringomyelia, possibly because the obex lies below the obstructing tonsils. ${ }^{219}$

The level of the cerebellar tonsils showed a similar association with presenting clinical features to those found in a recent MRI study. ${ }^{17}$ Features referable to the medulla oblongata were mainly difficulties with swallowing and phonation, and those referable to brainstem cerebellar connections were mainly ataxia and nystagmus. These, together with peripheral motor system signs, appeared significant because of their relatively high frequency in patients with severe tonsillar descent (group C). The relationship with peripheral sensory symptoms was mainly due to the high frequency in patients with moderate tonsillar descent (group B), and there was a close relationship between peripheral sensory disturbances and radiological signs of syringomyelia. It was no surprise perhaps to have found that neck pain appeared a significant feature in the presentation of mild but not severe malformations, the latter being associated with more disabling clinical manifestations.

In this study, important associations also were found between tonsillar descent and operative outcome. Unfavourable outcomes were more frequent with increasing tonsillar descent. With severe descent (group C) only $12 \%$ were improved and $69 \%$ had deteriorated on late assessment, whereas with mild descent (group A) only $18.5 \%$ had deteriorated. Clinical features least likely to improve were those related to brainstem/cerebellar connections and medulla oblongata, especially ataxia, as was also the case in the recent report by Menezes' group. ${ }^{14}$ The difference between outcomes on late assessment in group A and B was not significant, so once again it was group $\mathrm{C}$ which was different.

Outcome of both preoperative cough and posture related headache showed no relation to tonsillar descent or any other imaging parameter including size of cisterna magna, yet the latter feature was significantly improved in $62.6 \%$ cases. Posture and cough related headache, like drop attacks, are thought to result from intermittent tonsillar impaction in the foramen magnum ${ }^{27}$ which suboccipital craniectomy and duroplasty usually relieve very well. ${ }^{26}$ Therefore the lack of association of such features with small or obliterated cisterna magna or low lying tonsils both pre and postoperatively suggests that the origin of these symptoms is more complex.

Small size or obliteration of the cisterna magna, like increasing tonsillar descent, was related to clinical features localisable to the medulla oblongata and brainstem cerebellar connections. The lack of a definite relation between the size of the cisterna magna and other clinical features which were related to level of the tonsils, such as operative outcome, suggests that the level of tonsils is the more important of these interrelated parameters. It was surprising that no association was found between the size of the cisterna magna and syringomyelia, but once again the explanation in this study lies in group $C$ where the cisterna magna usually was small or obliterated and syringomyelia uncommon. However in a separate study using MRI, a lack of a relation between syringomyelia and the size of the CSF pathways across the foramen magnum also was observed. ${ }^{25}$

Opacification of the fourth ventricle with water soluble contrast medium nearly always occurs during examination of the craniovertebral junction in normal patients. It is a feature which cannot be determined using MRI and was of potential interest in view of theories still current about the causation of Chiari associated syringomyelia. It was recorded in only about $30 \%$ in this study which probably was a significant departure from normal. The maximum frequency of filling occurred in group B, which was the group with the highest prevalence of syringomyelia. This was surprising perhaps, since it is widely accepted that obstruction of the outlets of the fourth ventricle and cisterna magna by ectopic cerebellar tonsils and arachnoiditis are central to 
the development of syringomyelia, ${ }^{28-32}$ and strategies to correct this underpin operative treatment. Once again we believe the explanation in this study was simply the low incidence of fourth ventricle filling and of syringomyelia in patients of group $C$, because the differences between groups $A$ and $B$ were not significant. This probably also is the main reason why patients with filling of the fourth ventricle and an enlarged spinal cord had a better operative outcome than those without, because most of the former were in groups A and $B$.

Medullary compression could be evaluated adequately in only a small cohort of patients and we did not attempt to relate this to other radiological variables such as level of the tonsils. Although multivariate analysis would be required to distinguish the roles of this, and other features in this study in relation to outcome, it did seem to be related to one clinical feature, namely motor peripheral signs, but only when compression was severe. There seemed to be no relationship with syringomyelia which differed from the conclusions of Spinos et $\mathrm{al}^{24}$ but was similar to those of Clifton et $a l,{ }^{25}$ both based on MRI. It was expected that severe medullary compression also may have been relevant to some poor operative outcomes, which would be compatible with the known significance of severe spinal cord compression in cervical spondylosis $^{33}$ and atlanto-axial subluxation, ${ }^{34}$ but numbers of patients in this study were too small to permit any firm conclusion. The major compression usually was anterior, where it was likely to be made worse by flexing the head to facilitate a satisfactory posterior operative approach. ${ }^{35}$

\section{Conclusion}

The main outcome of this analysis has been to identify patients with tonsillar descent below the upper border of the neural arch of $\mathrm{C} 2$ as a special group. They had the highest frequency of disabling ataxia and nystagmus, the lowest frequency of syringomyelia, and suboccipital decompression was most likely to result in deterioration rather than improvement. On the other hand patients with the most favourable operative outcomes were those with mild tonsillar descent and a distended cervical syrinx. Clinical malfunction in the cerebellar/brainstem connections probably is related to the severity of the hind brain anomaly, and when the anomaly is severe, clinical features are most likely to be endpoints. This conclusion is supported by continuing observations at our institution where MRI virtually is now the only imaging modality used in preoperative assessment of this condition.

1 Paul KS, Lye RH, Strang FA, Dutton J. Arnold Chiari malformation. Review of 71 cases. $\mathcal{F}$ Neurosurg 1983;58: $183-7$.

2 Mohr PD, Strang FA, Sambrook MA, Boddie HG. Clinical and surgical features in 40 patients with pri-
mary cerebellar ectopia (Adult Chiari malformation). mary cerebellar ectopia
$Q \mathcal{F}$ Med $1977 ; 181: 85-96$
3 Dobkin BH. Syncope in the adult Chiari anomaly. Neurology 1978;28:718-20.

4 Gordon D. Neurological syndromes associated with craniovertebral anomalies. Proc $R$ Soc Med 1969;62:725-6.

5 Bronstein AM, Miller DH, Rudge P, Kendall BE. Down beating nystagmus: magnetic resonance imaging and beating nystagmus: magnetic resonance imaging

6 Caetano de Barros M, Farias W, Ataide L, Lins S. Basilar impression and Arnold Chiari malformation. A study of 66 cases. I Neurol Neurosurg Psychiatry 1968;31 596-605

7 Aboulezz AD, Sartor K, Geyer CA, Gado MH. Position of cerebellar tonsils in the normal population and in patients with Chiari malformation: a quantitative approach with MR imaging. F Comput Assis Tomogr 1985;9:1033-6.

8 Banerafi NK, Millar JHD. Chiari malformation presenting in adult life. The relationship to syringomyelia. Brain 1974;97:157-68.

9 Saez R, Onofrio B, Yanagihara T. Experience with Arnold Chiari malformation, 1960-1970. $尹$ Neurosurg 1976;45: 416-22.

10 Garcia-Uria J, Leunda G, Carrillo R, Bravo G. Syringomyelia: long-term results after posterior fossa decompression. F Neurosurg 1981;54:380-3.

11 Logue V, Edwards MR. Syringomyelia and its surgical treatment; an analysis of 75 patients. $\mathcal{F}$ Neurol Neurosurg Psychiatry 1981;44:273-85.

12 Di Lorenzo N, Aldo F, Guidetti B. Craniovertebral junction malformations. Clinico-radiological findings, longterm results and surgical indications in 63 cases. f Neurosurg 1982;57:603-8.

13 Eisenstat DDR, Bernstein M, Fleming JFR, et al. Chiari malformation in adults. A review of 40 cases. Can $\mathcal{J}$ Neurol Sci 1986;13:221-8.

14 Dyste SN, Menezes AH, Van Gilder JC. Symptomatic Chiari malformations. An analysis of presentation, management and longterm outcome. $\mathcal{F}$ Neurosurg 1989;71: agement

15 Curnes JT, Oakes WJ, Boyko OB. MR imaging of hindbrain deformity in Chiari II patients with and without symptoms of brainstem compression. AfNR 1989;10: 293-302.

16 Wolpert SM, Scott RM, Platenberg C, Runge VM. The clinical significance of hindbrain herniation and deformity as shown on MR images of patients with Chiari II malformations. AfNR 1988;9:1075-8.

17 Elster AD, Chen MY, Chiari I malformations: clinical and radiological re-appraisal. Radiology 1992;183:347-53.

18 Batzdorf V. Chiari I malformation with syringomyelia. ₹ Neurosurg 1988;68:726-30.

19 Rhoton AL. Microsurgery of the Arnold Chiari malformation in adults with and without hydrocephalus. f Neurosurg 1976;45:473-83.

20 O'Connor S, du Boulay G, Logue V. The normal position of the cerebellar tonsils as demonstrated by myelography. F Neurosurg 1973;39:387-9.

21 Baker H L. Myelographic examination of the posterior fossa with positive contrast medium. Radiology 1963; 81:791-801.

22 Stevens JM, Kendall E. Aspects of the anatomy of the cerebellum on computed tomography. Neuroradiology $1985 ; 27: 390-5$.

23 Naidich TP, McLone DS, Fulling KH. Chiari malformation: Part IV. The hindbrain deformity. Neuroradiology 1983;25:179-97.

24 Spinos E, Laster DW, Moody $\mathrm{P}$, et al. MR evaluation of the Chiari malformation at $0 \cdot 15 \mathrm{~T}$. $A \Re R \quad 1985 ; 144$ of the Chi $1143-8$.

25 Clifton AG, Stevens JM, Kendall BE. Idiopathic and Chiari associated syringomyelia in adults: observations on cerebrospinal fluid pathways at the foramen magnum using static MRI. Neuroradiology 1991;33 (suppl): 167-9.

26 Clifton A, Stevens JM, Kendall BE. Morphological features and their interrelationships in the adult Chiari malformation: an MRI study. Neuroradiology (in print).

27 Larson SJ, Sances A, Baker JB, Reigel DH. Herniated cerebellar tonsils and cough syncope. $\mathcal{f}$ Neurosurg 1974;40:524-8.

28 Williams B. Cerebrospinal fluid pressure changes in response to coughing. Brain 1976;99:331-46.

29 Barnett HJM, Foster JB, Hudgson P, eds. Syringomyelia. London: Saunders, 1973.

30 Newman PK, Terenty TR, Foster JB. Some observations on the pathogenesis of syringomyelia. $\mathcal{F}$ Neurol Neurosurg Psychiatry 1981;44:964-9.

31 Newton EJ. Syringomyelia as a manifestation of defective fourth ventricular drainage. Ann Roy Coll Surg Engl $1969 ; 44: 199-214$

32 du Boulay G, Shah S H, Currie J C, Logue V. The mechanisms of hydromyelia in Chiari type I malformations. Br F Radiol 1973;47:579-87.

33 Fujiwara K, Yenenobu K, Ebara S, Yamashita K, Ono K. The prognosis of surgery for cervical compression myelopathy. An analysis of the factors involved. $f$ Bone foint Surg 1989;71-B:393-8.

34 Hunter J, Stevens JM, Kendall BE, Moskovich R, Crockard HA. Radiological assessment for transoral surgery in rheumatoid arthritis, using dynamic computed myelography. Neuroradiology 1991;33 (suppl): puted $413-5$.

35 Brieg A. Biomechanics of the central nervous system. Some basic normal and pathological phenomena. Uppsala: Almquist and Wiksells, 1960 . 